\title{
CONTINUIDADE ESPACIAL DE CHUVAS INTENSAS NO ESTADO DE MINAS GERAIS
}

\author{
Spatial continuity of intense rainfall in Minas Gerais State, Brazil
}

\author{
Carlos Rogério de Mello ${ }^{1}$, Marcelo Ribeiro Viola², José Marcio de Melloº , Antônio Marciano da Silva ${ }^{1}$
}

\begin{abstract}
RESUMO
O mapeamento de variáveis climáticas, como chuvas intensas, é de fundamental importância para o manejo ambiental. Para isto, ferramentas estatísticas para interpolação espacial devem ser devidamente analisadas e caracterizadas. Assim, objetivou-se com este trabalho analisar modelos e métodos de modelagem do semivariograma que melhor se ajustem a chuvas intensas com duração de 20, 60, 360 e 1440 minutos e tempos de retorno de 5, 50 e 100 anos, fornecendo subsídios primordiais para espacialização da mesma pelo interpolador geoestatístico, para o Estado de Minas Gerais. Foram testados os modelos esférico, exponencial e gaussiano pelos métodos de ajuste da Máxima Verossimilhança (MV) e Mínimos Quadrados Ponderados (MQP). Utilizou-se como critério de escolha do melhor modelo, o menor erro médio gerado pela validação cruzada, e em caso de similaridade, também foram considerados o maior grau de dependência espacial e o menor efeito pepita, além da análise visual do ajuste do modelo ao semivariograma experimental. O modelo exponencial se sobressaiu em nove das doze situações analisadas, o gaussiano em duas e o esférico em uma situação. Quanto aos métodos de ajuste, o MQP sobressaiu em todos os casos estudados, o que permite sugerir o modelo exponencial ajustado pelo método dos mínimos quadrados ponderados como sendo o mais adequado para o mapeamento da chuva intensa para as condições do Estado de Minas Gerais.
\end{abstract}

Termos para indexação: Estrutura de dependência espacial, chuva intensa, geoestatística.

\begin{abstract}
Climate variables mapping, as intense rainfall, is very important to environmental management. Although, statistical tools for spatial interpolation should be analyzed and characterized. This paper aims to analyze models and methods of semi-variogram modeling applied to intense rainfall with duration time of 20, 60, 360 and 1440 minutes and 5, 50 and 100 years of recurrence and consequently, giving support for its mapping, using kriging, in Minas Gerais State. Exponential, Spherical and Gaussian semivariogram models were tested based on Weighted Minimum Square (WMS) and Maximum Likelihood (ML) methods, using GeoR software. For the best model and method evaluation was considered the mean absolute error produced by cross-validation. For mean error similarity, it was considered the spatial degree of dependence and smaller nugget effect. Visual adjustment of semi-variogram was also analyzed to complete the selection. Exponential model was predominant in nine of twelve situations, followed by Gaussian model in two situations and Spherical for just one. Weighted Minimum Square was the best adjust method in all situations. These results have indicated the exponential model adjusted by Weighted Minimum Square to intense rainfall mapping for Minas Gerais State conditions.
\end{abstract}

Index terms: Spatial dependence structure, intense rainfall, geostatistics.

(Recebido em 22 de junho de 2006 e aprovado em 2 de abril de 2007)

\section{INTRODUÇÃO}

A análise de chuvas intensas consiste em um dos produtos mais aplicados da hidrologia. Seu uso está associado ao dimensionamento de obras hidráulicas para controle de cheias, abastecimento e condução de água para usos múltiplos, drenagem urbana e do solo e modelagem e controle da erosão do solo. Segundo Mello et al. (2003b) quando a chuva intensa é associada a uma duração e a um tempo de retorno específico, passa a ser considerada como uma chuva de projeto, que uma vez aplicada a um modelo chuva-vazão, possibilita estimativa da vazão de projeto. Quando se necessita da chuva de projeto para localidades que não dispõem de séries históricas de precipitação, muitas vezes são utilizadas equações de locais próximos, o que pode comprometer a confiabilidade da estimativa.

Neste contexto, um dos principais ramos de pesquisa em hidrologia e climatologia consiste da aplicação do geoprocessamento, por meio da análise de técnicas para uma melhor interpolação espacial da chuva intensa, gerando mapas com boa aplicabilidade aos projetos. Neste

\footnotetext{
${ }^{1}$ Engenheiros Agrícola, Doutores, Professores - Departamento de Engenharia/DEG - Universidade Federal de Lavras/UFLA - Cx. P. 3037 - $37200-000$ crmello@ufla.br; marciano@ufla.br - Bolsista CNPq

²Engenheiro Agrícola, Mestre - Departamento de Engenharia/DEG - Universidade Federal de Lavras/UFLA-Cx. P. 3037-37200-000-m.r.viola@hotmail.com ${ }^{3}$ Engenheiro Florestal, Doutor, Professor - Departamento de Ciências Florestais/DCF - Universidade Federal de Lavras/UFLA - Cx. P. 3037 - $37200-000$ josemarcio@ufla.br
} 
intuito, Mello et al. (2003a) avaliaram 2 interpoladores distintos para os parâmetros da equação de chuvas intensas em localidades no estado de São Paulo, sendo o inverso do quadrado da distância e a krigagem geoestatística. Apesar dos autores não terem estudado o melhor método e modelo, e tendo trabalhado com o modelo exponencial ajustado pelo método da tentativa e erro ("a sentimento"), concluíram que a krigagem apresenta resultados consideravelmente mais precisos e que deve ser aplicada quando possível.

Mello et al. (2005) e Ribeiro Junior \& Diggle (2001) também relatam a supremacia do interpolador geoestatístico, em suas respectivas áreas de estudo. No entanto, esta condição está associada à modelagem do melhor semivariograma possível, acompanhado de uma análise exploratória prévia dos dados.

Assim, conduziu-se este trabalho com o objetivo do estudo da estrutura de dependência espacial da chuva intensa, associada a diferentes tempos de duração e de retorno, visando a subsidiar o mapeamento da chuva de projeto por krigagem geoestatística, no estado de Minas Gerais.

\section{MATERIAL E MÉTODOS}

\section{Caracterização da região de estudo e levantamento dos dados}

O estado de Minas Gerais encontra-se na região Sudeste do Brasil, possuindo grande variabilidade climática: clima Cwb/Cwa, característico da região Sul/ Sudeste do estado, influenciada pela Serra da Mantiqueira, com invernos frios e verões brandos e chuvosos, Aw na região Triângulo Mineiro, Leste e norte do Estado, com invernos brandos e verões quentes, além de concentração das chuvas no verão, e clima BSw na região do Vale do Jequitinhonha, com características semi-áridas (ANTUNES, 1986).

Foram utilizadas neste trabalho séries históricas de intensidade máxima de precipitações, associadas às durações de 20, 60, 360 e 1440 minutos, geradas por Freitas et al. (2001), para 177 localidades no estado de Minas Gerais. A estas séries foi ajustada e aplicada a distribuição de Gumbel, segundo Haan (2002) para estimativa de cada um dos valores associados aos tempos de retorno de 5, 50 e 100 anos, totalizando 12 diferentes situações para a chuva de projeto.

\section{Análise Espacial dos dados}

A fim de se obter um mapa de Minas Gerais georreferenciado em coordenadas métricas, livre de fusos- horários, adotou-se a projeção Cônica Albers Equivalente, uma vez que, segundo Dieulin et al. (2006), essa projeção é a mais indicada para utilização em Sistemas de Informações Geográficas (SIG), pois possui a característica de preservar a área.

A análise geoestatística deve ser precedida da análise exploratória dos dados, com o objetivo de se estruturar um estudo mais consistente, de forma que algumas pressuposições geoestatísticas possam ser atendidas, especialmente a não tendenciosidade. $\mathrm{O}$ programa GeoR, de livre acesso, foi utilizado em todas as etapas do trabalho, inclusive na análise exploratória, que constituiu-se na geração de gráficos de tendência, histogramas de frequiência das diferenças entre pares de pontos e análise de "outliers" por meio de gráficos "boxplot", com o objetivo de se verificar se há ou não tendência dos dados e retirá-la quando constatada, para aplicação dos conceitos previstos na geoestatística (RIBEIRO JUNIOR \& DIGGLE, 2001).

Para cada situação de chuva intensa foi desenvolvido um estudo da continuidade espacial, avaliando os modelos esférico, exponencial e gaussiano, cujas estruturas matemáticas e descrição de cada parâmetro estão apresentados em Isaack \& Srivastava (1989) e Mello (2004).

\section{Métodos de Ajuste de Semivariogramas}

O ajuste do modelo espacial ao semivariograma experimental é desenvolvido por métodos matemáticos, estimando-se os parâmetros característicos de cada modelo. Neste trabalho foram testados os métodos da Máxima Verossimilhança (MV) e Mínimos Quadrados Ponderados (MQP). Segundo Mello (2004), o método MQP é uma tentativa de ponderar a semivariância, uma vez que a ponderação pela variância das estimativas das semivariâncias não é conhecida, o que é obtido dividindose o erro quadrático médio pelo número de pares de pontos em cada distância no semivariograma.

A MV denota a máxima probabilidade de algo ser verdadeiro. Este estimador apresenta propriedades tais como ser assintoticamente não tendencioso, fornecer estimativas de variância mínima, ser consistente e invariante (RIBEIRO JUNIOR \& DIGGLE, 2001). Este método atua maximizando a função de densidade de probabilidades em relação aos efeitos fixos e aos componentes de variância dos efeitos aleatórios do modelo, sempre fornecendo estimativas positivas de componentes de variância. Ressalta-se que o método da MV ajusta-se aos dados amostrados e não aos pontos do semivariograma e é imprescindível que haja normalidade das diferenças entre 
os pontos amostrados, conhecida como multivariada normal (MELLO, 2004).

\section{Escolha do método e modelo de semivariograma}

A fim de verificar a qualidade do ajuste do semivariograma aos dados experimentais, e assim constatar o modelo que melhor se adequou, foram avaliados 2 parâmetros, ambos intimamente ligados à geoestatística: a Validação Cruzada ou auto-validação e o grau de dependência espacial, ambos segundo Mello et al. (2005). A Validação Cruzada consiste em estimar, com base no modelo de semivariograma ajustado, os pontos que foram realmente amostrados, podendo-se assim, comparar o valor estimado ao real. $\mathrm{O}$ procedimento consiste em eliminar cada um dos pontos amostrados e estimar, por meio da krigagem, o valor para o local amostrado, com base nos demais pontos. Assim, trabalhou-se com os erros médio e máximo produzidos pelos métodos e modelos trabalhados.

$\mathrm{O}$ grau de dependência (GD) consiste da razão entre a variância estrutural $\left(\mathrm{C}_{1}\right)$ e o patamar $\left(\mathrm{C}_{0}+\mathrm{C}_{1}\right)$ e permite classificar o grau de dependência espacial. Segundo Mello (2004), este parâmetro pode ser calculado por:

$$
\mathrm{GD}=\left(\frac{\mathrm{C}_{1}}{\mathrm{C}_{\mathrm{o}}+\mathrm{C}_{1}}\right) \times 100 \text { onde: }
$$

$\mathrm{C}_{0}$ : efeito pepita (representa a variação aleatória do fenômeno estudado); $C_{1:}$ Variância estruturada (variância explicada pela componente espacial); $\mathrm{C}_{0}+\mathrm{C}_{1}$ : patamar (variação total do fenômeno avaliado).

A classificação adotada é a seguinte: GD £ $25 \%$ (grau de dependência fraco), entre 25 e $75 \%$ (moderado) e $>75 \%$ (forte), conforme Cambardella et al. (1994). Basicamente, este parâmetro de análise consiste de um avaliador complementar aos erros gerados pela validação cruzada e foi tratado desta forma, neste trabalho. Além disto, em caso de igualdade dos erros e GD, foi considerado aquele com o menor efeito pepita.

\section{RESULTADOS E DISCUSSÃO}

\section{Análise Exploratória dos Dados}

Na Figura 1 apresentam-se gráficos de tendência dos dados de chuva intensa e histograma da diferença entre pares de pontos para a situação de tempo de duração de 20 minutos e tempo de retorno de 5 anos. As demais situações analisadas proporcionaram comportamento semelhante tanto em termos de tendência quanto de normalidade das diferenças. Analisando o comportamento da distribuição dos dados nas direções Norte-Sul (Latitude) e Leste-Oeste (Longitude), constata-se boa distribuição dos mesmos, o que caracteriza não tendenciosidade dos dados, atendendo às condições para que a hipótese intrínseca seja aplicada.

O histograma das diferenças entre pares de dados (Figura 1c) permite uma boa inferência sobre a existência da distribuição multivariada normal, que é uma condição desejável em geoestatística, particularmente no método da máxima verossimilhança (MV), o qual pressupõe esta condição (RIBEIRO JUNIOR \& DIGGLE, 2001). Com isto, analisando-se o histograma da Figura 1c, constata-se boa distribuição de frequiência em termos de normalidade das diferenças, permitindo verificar existência de normalidade multivariada, significando que os ajustes pelo método da Máxima Verossimilhança podem ser possíveis. O gráfico "boxplot" permite estudar a simetria das distribuições e detectar os "outliers", os quais constituem-se de pontos amostrados no espaço cujos valores são discrepantes dos demais. Os dados discrepantes podem afetar a variância aleatória existente no processo, implicando na presença ou ausência de correlação espacial (RIBEIRO JÚNIOR \& DIGGLE, 2001). Portanto estes pontos foram removidos para posterior análise espacial.

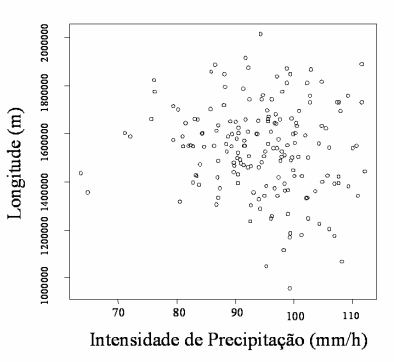

(a)

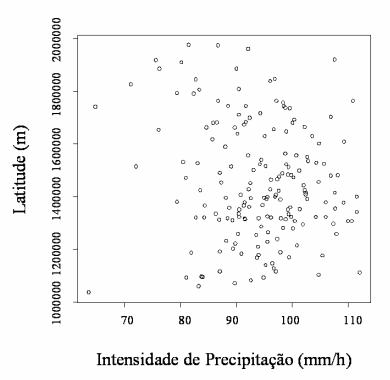

(b)

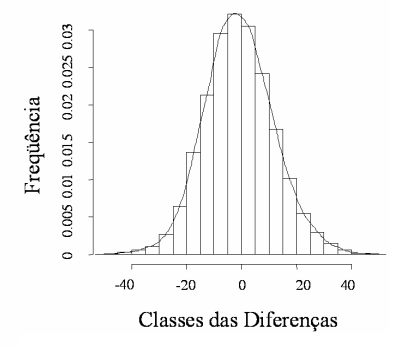

(c)

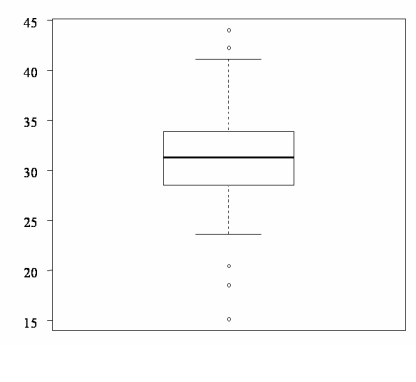

(d)

Figura 1 - Gráficos de tendência (a, b), histograma das diferenças (c) entre pares de pontos e "boxplot" (d), para chuva intensa com tempo de duração de 20 minutos e de retorno 5 anos. 


\section{Modelos e métodos de ajuste de semivariogramas}

O comportamento dos modelos de semivariograma ajustados pelos métodos MV e MQP pode ser analisado por meio do grau de dependência espacial (GD) e erros médio e máximo, gerados pela auto-validação. Na Tabela 1, apresentam-se os resultados da auto-validação, grau de dependência espacial e parâmetros dos semivariogramas ajustados, com a indicação do melhor método e modelo (em negrito), de acordo com os critérios adotados.

$\mathrm{Na}$ maioria dos eventos estudados a diferença entre os erros dos modelos ajustados foi pequena, levando-se a adotar como critério decisivo para escolha o maior grau de dependência espacial. Entretanto, especificamente para a chuva intensa de 60 minutos tanto os erros quanto o grau de dependência, foram muito próximos, resultando na escolha do modelo e método que produziram o menor efeito pepita. Das doze situações estudadas, o modelo exponencial ajustado pelo método dos mínimos quadrados ponderados (MQP) se sobressaiu em nove, o modelo gaussiano em duas e o esférico em uma situação (Tabela 1). Observa-se que, para o tempo de duração de 20 minutos, houve maior dificuldade de ajuste dos semivariogramas pelo MQP, especialmente para o modelo exponencial, tendo sido observado, em duas situações, efeito pepita puro para este modelo e método. Convém ressaltar que houve dificuldade de ajuste do modelo para o tempo de retorno de 100 anos, tendo sido encontrado, da mesma forma, efeito pepita puro. Neste caso excepcionalmente, optou-se por adotar o tempo de retorno de 20 anos (Tabela 1).

Analisando o desempenho geral dos modelos e métodos de ajuste dos semivariogramas, fica evidente a superioridade do modelo exponencial (independentemente do método) e do método dos Mínimos Quadrados Ponderados (independentemente dos modelos) para os dados de chuvas intensas no estado de Minas Gerais, uma vez que esta metodologia prevaleceu em todos os casos estudados neste trabalho. No entanto, para tempo de duração de 20 minutos, o método MQP apresentou efeito pepita puro para quatro diferentes situações (duas com modelo exponencial, uma com modelo esférico e uma com o modelo gaussiano), enquanto que o método da Máxima Verossimilhança (MV) não produziu este resultado em nenhuma situação. Isto ocorreu porque o ajuste do modelo pela MV foi favorecido pela condição de normalidade multivariada dos dados. Assim, o método MV pode ser aplicado para validar o ajuste produzido, especialmente quando da ocorrência de efeito pepita puro pelo método MQP.

Carvalho et al. (2004) estudando a precipitação pluvial média anual no estado de São Paulo, utilizaram dados de 379 estações pluviométricas, visando a testar a adequabilidade do emprego do método MQP para ajuste de modelos ao semivariograma em comparação ao método de tentativa e erro ("a sentimento") averiguou utilizando o método de auto-validação "jack knifing", superioridade do método MQP. Ainda de acordo com este estudo, houve um melhor ajuste dos dados com o modelo exponencial frente ao esférico e gaussiano.

Na Figura 2 apresentam-se os semivariogramas selecionados para cada uma das situações estudadas, observando-se boa adequação dos modelos ao semivariograma experimental, reforçando as avaliações e resultados produzidos pela validação cruzada.

A literatura trata o grau de dependência espacial menor que $25 \%$ como fraco, entre 25 e $75 \%$ como moderado e maior que $75 \%$ como alto. O grau de dependência médio dos modelos escolhidos foi de 63,50\%, apresentando-se no nível moderado, porém próximo ao grau de alta dependência espacial. $\mathrm{O}$ evento que apresentou o maior grau de dependência foi 360 minutos e tempo de retorno de 5 anos $(66,78 \%)$ e o que apresentou o menor, foi 20 minutos e tempo de retorno de 50 anos $(53,32 \%)$.

Em relação ao erro médio (E.M) encontrou-se uma amplitude de 7,07\% (precipitação de 20 minutos para um tempo de retorno de 5 anos) a 13,46\% (precipitação de 360 minutos de duração para tempo de retorno de 100 anos), dentre os modelos escolhidos, apresentando uma média de 10,42\%. Segundo Mello et al. (2003a) e Vieira et al. (2002) estes erros são consideravelmente baixos, demonstrando o elevado potencial que a interpolação geoestatística (krigagem) possui para o mapeamento da chuva intensa e por consequiência, a chuva de projeto. $\mathrm{O}$ interpolador que não considerar a estrutura de dependência espacial, certamente produzirá estimativas tendenciosas com mapas interpolados com valores viesados, distorcendo a realidade.

O comportamento do alcance do semivariograma está associado à existência de estrutura de dependência espacial e aplicação dos princípios da correlação espacial. Estes variaram aproximadamente entre 150 e $1200 \mathrm{~km}$, mostrando a extensão da estrutura de dependência espacial da variável chuva intensa, que em alguns casos, cobre praticamente todo o estado. A questão central do alcance consiste do uso dos conceitos da estatística clássica e da estatística espacial. Pelos resultados foi possível demonstrar que, em se tratando de chuvas intensas, os conceitos da estatística espacial propiciam resultados mais precisos, quando comparado com a estatística clássica. Resultados demonstrando o ganho de precisão com estatística espacial na presença de correlação, podem ser vistos em Mello et al. (2003a, 2005), que trabalharam, respectivamente, com volume de madeira de eucalipto e chuvas intensas para o Estado de São Paulo. Estimativas precisas resultam em mapas de krigagem com alta qualidade. 
Tabela 1 - Parâmetros dos modelos de semivariograma $\left(\mathrm{C}_{0}\right.$ - efeito pepita; $\mathrm{C}_{1}$ - variação estruturada; $\mathrm{C}_{1}-\mathrm{C}_{0}-$ patamar; A - Alcance; GD - grau de dependência; E.M - erro médio; E.Ma - erro máximo) ajustados para chuvas intensas em função de diferentes tempos de duração e retorno.

\begin{tabular}{|c|c|c|c|c|c|c|c|}
\hline Modelo & Método & $\mathrm{C}_{0}$ & $\mathrm{C}_{1}-\mathrm{C}_{0}$ & $\mathrm{~A}(\mathrm{~km})$ & GD & E.M & E.Ma \\
\hline \multicolumn{8}{|c|}{ Chuva intensa de 20 minutos e TR de 5 anos } \\
\hline Exp. & M.V & 60,16 & 22,24 & 450,00 & 57,80 & 7,04 & 33,24 \\
\hline Esf. & M.V. & 63,37 & 19,20 & 400,00 & 56,57 & 7,10 & 33,10 \\
\hline Gau. & M.V. & 67,28 & 27,57 & 400,00 & 58,50 & 7,01 & 32,84 \\
\hline Exp. & M.Q.P. & EPP & EPP & EPP & EPP & EPP & EPP \\
\hline Esf. & M.Q.P. & EPP & EPP & EPP & EPP & EPP & EPP \\
\hline Gau. & M.Q.P. & 62,92 & 44,43 & 513,77 & 63,05 & 6,99 & 32,75 \\
\hline \multicolumn{8}{|c|}{ Chuva intensa de 20 minutos e TR de 20 anos } \\
\hline Exp. & M.V & 140,65 & 28,93 & 1200,00 & 54,66 & 7,78 & 36,74 \\
\hline Esf. & M.V. & 141,40 & 17,10 & 400,00 & 52,85 & 7,80 & 36,57 \\
\hline Gau. & M.V. & 145,91 & 21,98 & 400,00 & 53,50 & 7,78 & 36,83 \\
\hline Exp. & M.Q.P. & 122,68 & 60,77 & 1200,30 & 59,95 & 7,79 & 35,98 \\
\hline Esf. & M.Q.P. & 126,07 & 35,97 & 400,10 & 56,24 & 7,82 & 35,83 \\
\hline Gau. & M.Q.P. & 139,74 & 135,02 & $\mathbf{1 0 9 7 , 8 0}$ & 66,29 & $\mathbf{7 , 8 1}$ & $\mathbf{3 7 , 8 5}$ \\
\hline \multicolumn{8}{|c|}{ Chuva intensa de 20 minutos e TR de 50 anos } \\
\hline Exp. & M.V & 218,15 & 17,09 & 450,00 & 51,88 & 8,40 & 39,12 \\
\hline Esf. & M.V. & 221,81 & 12,74 & 400,00 & 51,40 & 8,41 & 39,35 \\
\hline Gau. & M.V. & 220,23 & 13,51 & 136,36 & 51,49 & 8,41 & 39,33 \\
\hline Exp. & M.Q.P. & EPP & EPP & EPP & EPP & EPP & EPP \\
\hline Esf. & M.Q.P. & 209,08 & 29,72 & 400,29 & 53,32 & $\mathbf{8 , 4 0}$ & 38,74 \\
\hline Gau. & M.Q.P. & EPP & EPP & EPP & EPP & EPP & EPP \\
\hline \multicolumn{8}{|c|}{ Chuva intensa de 60 minutos e TR de 5 anos } \\
\hline Exp. & M.V & 27,94 & 19,25 & 1200,00 & 62,81 & 8,15 & 51,26 \\
\hline Esf. & M.V. & 28,57 & 10,97 & 400,00 & 58,05 & 8,22 & 51,20 \\
\hline Gau.. & M.V. & 31,23 & 14,91 & 400,00 & 59,64 & 8,25 & 50,62 \\
\hline Exp. & M.Q.P. & 21,87 & 29,07 & 1200,00 & 69,96 & 8,15 & 51,82 \\
\hline Esf. & M.Q.P. & 18,94 & 22,59 & 400,08 & 68,68 & 8,22 & 52,17 \\
\hline Gau. & M.Q.P. & 28,15 & 18,95 & 400,01 & 62,59 & 8,26 & 50,80 \\
\hline \multicolumn{8}{|c|}{ Chuva intensa de 60 minutos e TR de 50 anos } \\
\hline Exp. & M.V & 93,31 & 41,52 & 1200,00 & 59,10 & 9,89 & 46,44 \\
\hline Esf. & M.V. & 94,96 & 22,46 & 400,00 & 55,29 & 9,98 & 46,41 \\
\hline Gau.. & M.V. & 102,04 & 25,40 & 400,00 & 55,53 & 10,01 & 45,42 \\
\hline Exp. & M.Q.P. & $\mathbf{7 4 , 8 3}$ & 49,09 & 555,60 & 62,35 & $\mathbf{9 , 8 7}$ & 46,81 \\
\hline Esf. & M.Q.P. & 77,34 & 42,93 & 400,05 & 60,86 & 9,94 & 47,46 \\
\hline Gau. & M.Q.P. & 96,93 & 31,93 & 400,04 & 57,07 & 10,01 & 45,67 \\
\hline \multicolumn{8}{|c|}{ Chuva intensa de 60 minutos e TR de 100 anos } \\
\hline Exp. & M.V & 122,95 & 48,62 & 1200,00 & 58,25 & 10,42 & 45,50 \\
\hline Esf. & M.V. & 124,84 & 26,35 & 400,00 & 54,77 & 10,42 & 45,50 \\
\hline Gau.. & M.V. & 133,53 & 28,58 & 400,00 & 54,83 & 10,54 & 44,42 \\
\hline Exp. & M.Q.P. & 93,54 & 63,69 & 450,30 & 62,70 & 10,41 & 45,84 \\
\hline Esf. & M.Q.P. & 104,55 & 50,01 & 40,07 & 59,65 & 10,46 & 46,56 \\
\hline Gau. & M.Q.P. & 127,86 & 36,24 & 400,06 & 56,21 & 10,53 & 44,69 \\
\hline
\end{tabular}

Continua... 
Tabela 1 - Continuação...

\begin{tabular}{|c|c|c|c|c|c|c|c|}
\hline Modelo & Método & $\mathrm{C}_{0}$ & $\mathrm{C}_{1}-\mathrm{C}_{0}$ & $\mathrm{~A}(\mathrm{~km})$ & GD & E.M & E.Ma \\
\hline \multicolumn{8}{|c|}{ Chuva intensa de 360 minutos e TR de 5 anos } \\
\hline Exp. & M.V & 2,42 & 2,01 & 1200,00 & 64,67 & 10,01 & 54,70 \\
\hline Esf. & M.V. & 2,48 & 1,11 & 400,00 & 59,23 & 10,06 & 53,76 \\
\hline Gau.. & M.V. & 2,75 & 1,67 & 400,00 & 61,66 & 9,96 & 53,46 \\
\hline Exp. & M.Q.P. & 1,34 & 1,35 & 450,00 & 66,78 & 10,16 & 53,60 \\
\hline Esf. & M.Q.P. & 1,57 & 1,07 & 400,00 & 62,75 & 10,17 & 52,58 \\
\hline Gau. & M.Q.P. & 2,20 & 1,81 & 243,84 & 64,60 & 10,14 & 52,65 \\
\hline \multicolumn{8}{|c|}{ Chuva intensa de 360 minutos e TR de 50 anos } \\
\hline Exp. & M.V & 9,85 & 4,67 & 900,00 & 59,59 & 12,83 & 93,93 \\
\hline Esf. & M.V. & 9,66 & 3,30 & 300,00 & 57,30 & 12,82 & 93,70 \\
\hline Gau.. & M.V. & 10,84 & 2,78 & 300,00 & 55,68 & 12,82 & 94,01 \\
\hline Exp. & M.Q.P. & 4,15 & 5,07 & 150,00 & 68,96 & 13,08 & $\mathbf{8 8 , 9 7}$ \\
\hline Esf. & M.Q.P. & 6,09 & 7,57 & 300,16 & 69,16 & 13,17 & 88,17 \\
\hline Gau. & M.Q.P. & 8,35 & 5,72 & 205,30 & 62,76 & 12,95 & 89,93 \\
\hline \multicolumn{8}{|c|}{ Chuva intensa de 360 minutos e TR de 100 anos } \\
\hline Exp. & M.V & 9,06 & 2,68 & 300,00 & 56,44 & 13,45 & 100,88 \\
\hline Esf. & M.V. & 8,54 & 3,16 & 300,00 & 57,80 & 13,45 & 100,49 \\
\hline Gau.. & M.V. & 9,53 & 2,19 & 100,00 & 55,16 & 13,46 & 101,17 \\
\hline Exp. & M.Q.P. & 7,96 & 4,46 & 300,00 & 60,94 & 13,46 & 95,08 \\
\hline Esf. & M.Q.P. & 9,33 & 2,77 & 300,00 & 56,31 & 13,85 & 93,35 \\
\hline Gau. & M.Q.P. & 10,94 & 1,35 & 300,00 & 52,89 & 13,59 & 96,29 \\
\hline \multicolumn{8}{|c|}{ Chuva intensa de 1440 minutos e TR de 5 anos } \\
\hline Exp. & M.V & 0,28 & 0,17 & 1125,00 & 61,35 & 10,35 & 52,12 \\
\hline Esf. & M.V. & 0,28 & 0,11 & 375,00 & 58,27 & 10,42 & 52,28 \\
\hline Gau.. & M.V. & 0,31 & 0,10 & 375,00 & 56,89 & 10,49 & 52,44 \\
\hline Exp. & M.Q.P. & 0,17 & 0,18 & 450,00 & 67,36 & 10,30 & 51,93 \\
\hline Esf. & M.Q.P. & 0,25 & 0,17 & 375,00 & 62,86 & 10,36 & 52,06 \\
\hline Gau. & M.Q.P. & 0,30 & 0,17 & 375,00 & 61,19 & 10,53 & 52,47 \\
\hline \multicolumn{8}{|c|}{ Chuva intensa de 1440 minutos e TR de 50 anos } \\
\hline Exp. & M.V & 0,66 & 0,24 & 300,00 & 57,62 & 12,90 & 52,92 \\
\hline Esf. & M.V. & 0,63 & 0,28 & 100,00 & 59,20 & 12,99 & 51,14 \\
\hline Gau.. & M.V. & 0,71 & 0,19 & 100,00 & 55,77 & 12,96 & 56,46 \\
\hline Exp. & M.Q.P. & 0,62 & $\mathbf{0 , 3 1}$ & 300,00 & 60,09 & 12,90 & 52,11 \\
\hline Esf. & M.Q.P. & 0,71 & 0,21 & 300,00 & 56,31 & 13,03 & 52,78 \\
\hline Gau. & M.Q.P. & 0,75 & 0,23 & 300,00 & 56,72 & 12,98 & 56,21 \\
\hline \multicolumn{8}{|c|}{ Chuva intensa de 1440 minutos e TR de 100 anos } \\
\hline Exp. & M.V & 0,95 & 0,26 & 450,00 & 56,06 & 13,47 & 55,77 \\
\hline Esf. & M.V. & 0,91 & 0,31 & 150,00 & 57,21 & 13,62 & 54,14 \\
\hline Gau.. & M.V. & 1,01 & 0,20 & 150,00 & 54,54 & 13,51 & 59,19 \\
\hline Exp. & M.Q.P. & 0,86 & 0,43 & 450,00 & 60,22 & 13,46 & 55,43 \\
\hline Esf. & M.Q.P. & 0,93 & 0,30 & 150,00 & 56,91 & 13,68 & 56,25 \\
\hline Gau. & M.Q.P. & 1,00 & 0,25 & 150,00 & 55,46 & 13,52 & 59,09 \\
\hline
\end{tabular}

EPP: Efeito Pepita Puro. 
MELLO, C. R. de et al.
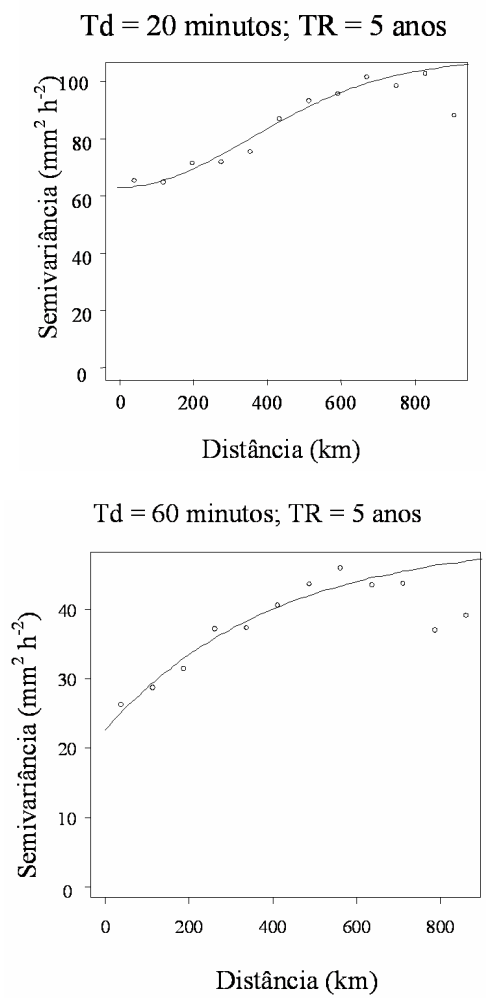

$\mathrm{Td}=360$ minutos; $\mathrm{TR}=\mathbf{5}$ anos

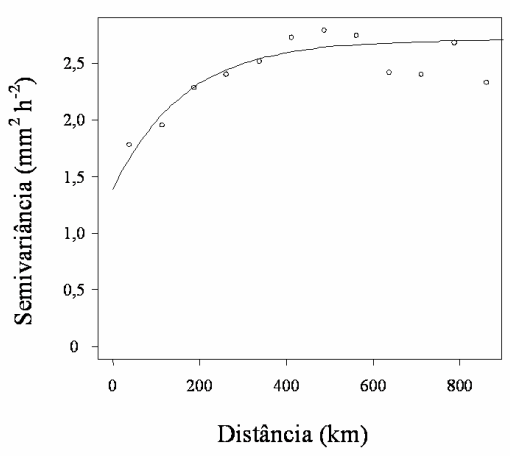

$\mathrm{Td}=1440$ minutos; $\mathrm{TR}=5$ anos

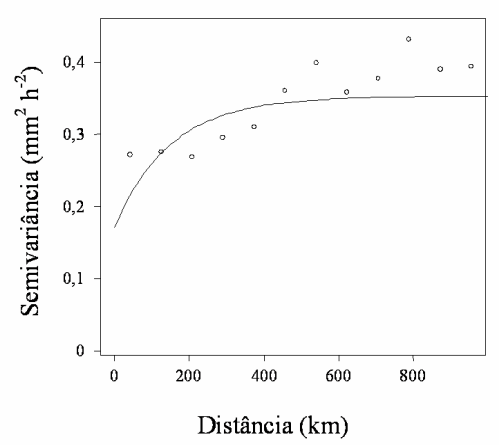

$\mathrm{Td}=20$ minutos; $\mathrm{TR}=20$ anos

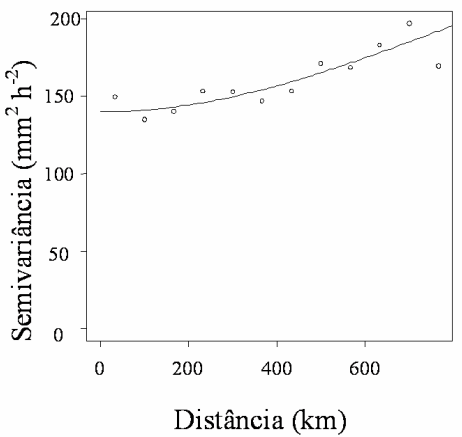

$\mathrm{Td}=60$ minutos; $\mathrm{TR}=50$ anos

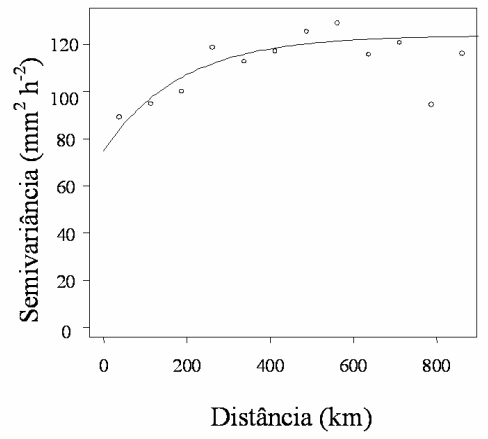

$\mathrm{Td}=360$ minutos; $\mathrm{TR}=50$ anos

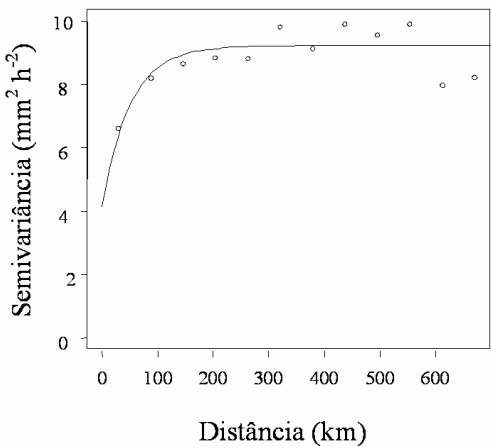

$\mathrm{Td}=1440$ minutos; $\mathrm{TR}=50$ anos

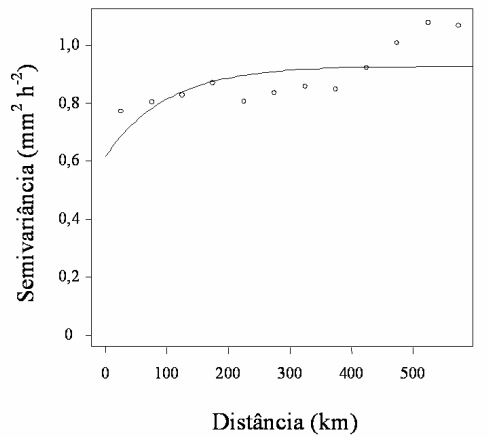

$\mathrm{Td}=20$ minutos; $\mathrm{TR}=50$ anos

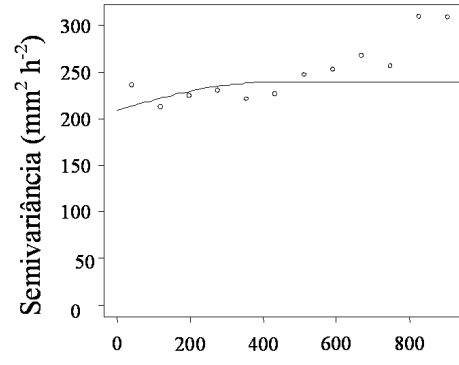

Distância $(\mathrm{km})$

$\mathrm{Td}=60$ minutos; $\mathrm{TR}=100$ anos

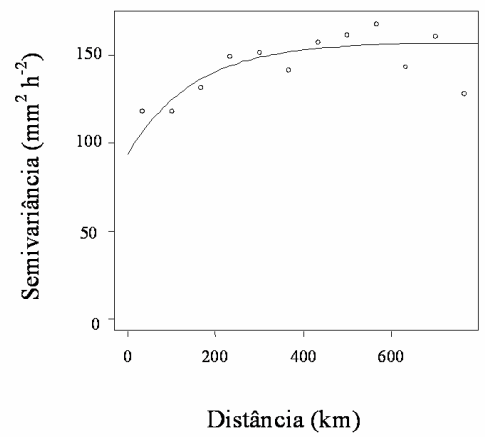

$\mathrm{Td}=360$ minutos; $\mathrm{TR}=100$ anos

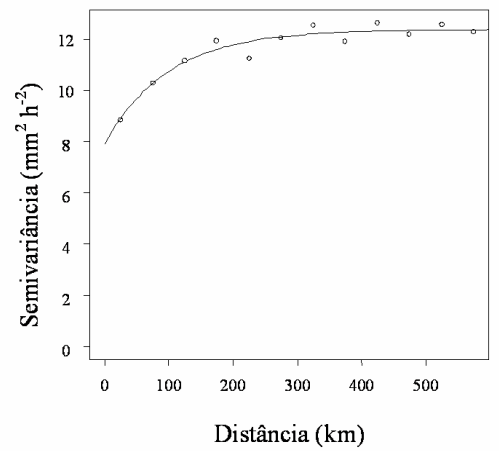

$\mathrm{Td}=1440$ minutos; $\mathrm{TR}=100$ anos

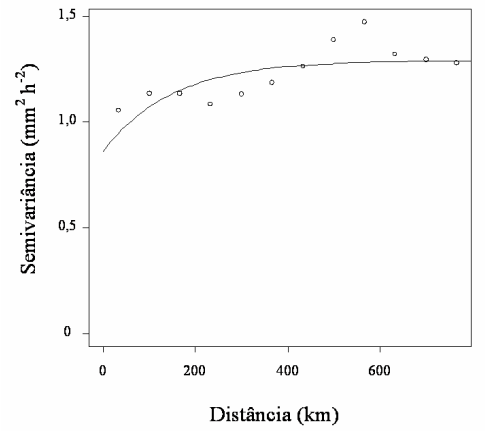

Figura 2 - Semivariograma adotado para cada situação de chuva intensa analisada. 


\section{CONCLUSÕES}

O modelo exponencial de semivariograma foi o que melhor se ajustou aos dados de chuvas intensas no estado de Minas Gerais, independentemente do método de ajuste;

O método dos Mínimos Quadrados Ponderados destacou-se em relação ao método da Máxima Verossimilhança, sendo recomendado para estudos associados à aplicação da geoestatística na espacialização da chuva intensa;

O erro médio produzido pela Validação Cruzada foi muito baixo, demonstrando o potencial da krigagem geoestatística para interpolação da chuva intensa;

Os ajustes do semivariogramas resultaram em graus de dependência espacial moderados, demonstrando que estes dados possuem estrutura de dependência espacial.

\section{REFERÊNCIAS BIBLIOGRÁFICAS}

ANTUNES, F. Z. Caracterização climática do estado de Minas Gerais. Informe Agropecuário, Belo Horizonte, v. 12, p. 9-17, 1986.

CAMBARDELLA, C. A.; MOORMAN, T. B.; NOVAK, J. M.; PARKIN, T. B.; KARLEN, D. L.; TURCO, R. F.; KONOPKA, A. E. Field scale variability of soil properties in central Iowa soils. Soil Science Society of America Journal, Madison, v. 58, n. 5, p. 1501-1511, 1994.

CARVALHO, J. R. P. de; VIEIRA, S. R.; VENDRUSCULO, L. G. Uso da técnica de mínimos quadrados ponderados para ajuste de modelos as semivariograma. Campinas: Embrapa Informática Agropecuária, 2004. (Boletim de pesquisa e desenvolvimento).

DIEULIN, C.; BOYER, J. F.; ARDOIN-BARDIN, S.; DEZETTER, A. The contribution of GIS to hydrological modeling. In: DEMUTH, S.; GUSTARD, A.; PLANOS, E.; SCATENA, F.; SERVAT, E. (Eds.). Climate variability and change: hydrological impacts. Wallingford: International Association of Hydrological Science, 2006. p. 66-74.

FREITAS, A. J.; SILVA, D. D.; PRUSKI, F. F.; PINTO, F. A.; PEREIRA, S. B.; GOMES FILHO, R. R.; TEIXEIRA, A. F.;
BAENA, L. G. N.; MELLO, L. T. A.;NOVAES, L. F.Equações de chuvas intensas no Estado de Minas Gerais. Belo Horizonte: Copasa; UFV, 2001. 65 p.

HAAN, C. T. Statistical methods in hydrology. 2. ed. Ames: The Iowa State University, 2002. 377 p.

ISAAK, E. H.; SRIVASTAVA, R. M. Applied geoestatistic: an introduction. New York: Oxford University, 1989. 561 p.

MELLO, C. R. de; LIMA, J. M.; SILVA, A. M.; MELLO, J. M.; OLIVEIRA, M. S. Krigagem e inverso do quadrado da distância para interpolação dos parâmetros da equação de chuvas intensas. Revista Brasileira de Ciência do Solo, Viçosa, v. 27, n. 5, p. 925-933, 2003 a.

MELLO, C. R. de; SILVA, A. M.; LIMA, J. M.; FERREIRA, D. F.; OLIVEIRA, M. S. Modelos matemáticos para predição da chuva de projeto para regiões do estado de Minas Gerais. Revista Brasileira de Engenharia Agrícola e Ambiental, Campina Grande, v. 7, n. 1, p. 121-128, 2003b.

MELLO, J. M.; BATISTA, J. L. F.; RIBEIRO JUNIOR, P. J.; OLIVEIRA, M. S. Ajuste e seleção de modelos espaciais de semivariograma visand à estimativa volumétrica de Eucaliptus grandis. Scientia Forestalis, Piracicaba, v. 1, n. 1, p. 25-37, 2005.

MELLO, J. M. de. Geoestatística aplicada ao inventário florestal. 2004. 110 f. Tese (Doutorado em Recursos Florestais) - Escola Superior de Agricultura de Luiz de Queiroz, Piracicaba, 2004.

RIBEIRO JUNIOR, P. P.; DIGGLE, P. P. GeoR: a package for geoestatistical analysis. R-News, Rochester, v. 1, n. 2, p. 15-18, 2001.

VIEIRA, S. R.; MILLETE, J.; TOPP, G. C.; REYNOLDS, W. D. Handbook for geostatistical analysis of variability in soil and climate data. In: NOVAIS, R. F.; SCHAEFER, C. E. G. R. (Eds.). Tópicos em ciência do solo. Viçosa: SBCS, 2002. v. 2, p. 1-46. 